\title{
CALCULATION OF THE FLUID FORCE ACTING ON THE BEARING JOURNAL IN THE STEADY-STATE POSITION
}

\author{
J. Tůma *, J. Šimek ${ }^{* *}$, M. Kozubková ${ }^{* * * *}$
}

\begin{abstract}
The paper relates to the fluid forces acting on the journal of the slide bearing. These forces can be calculated using the Reynolds equation. The development of actively controlled journal bearings with piezoactuators requires the model of the controlled system in the form of formulas that allow respecting the influence of all parameters. The paper provides a simplified solution to the Reynolds equation that justifies the stiffness matrix in the equivalent model suggested by Muszynska with the use of springs and dampers.
\end{abstract}

\section{Keywords: journal bearings, Reynolds equation, fluid forces, Muszynska model}

\section{Introduction}

An analysis of the behavior of active vibration control systems requires describing a controlled system with the use of the linear equations that are suitable for the calculation of the system transfer functions and the controller adjustment (Tůma et al., 2017). For this purpose, the approximate model proposed by Muszynska is sufficient. This analysis, however, is aimed at the calculation of the stiffness matrix of the motion equation with the use of the Reynolds equation to estimate the behavior of the journal bearing at the extra high rotational speed. Instead of numerical integration of the Reynolds equation, the derivation of the formulas to calculate the model parameters is used.

\section{2.}

The bearing journal can be considered as a rigid body rotating within the bearing housing at an angular velocity $\Omega$. For simplicity, it is assumed that the rotation axis does not change its direction. Fluid forces are caused by the hydrodynamic pressure generated in the oil film, whose total mass relative to the journal and rotor is negligible. The oil pumped by the rotating journal surface produces an oil wedge that lifts up the bearing journal so that it does not touch the inner walls of the housing. The coordinate system of a plain cylindrical journal bearing is shown on the left side in Fig. 1. The planar motion of the bearing journal at the $x$ and $y$ coordinates can be described by two motion equations arranged into a matrix equation

$$
\left[\begin{array}{cc}
M & 0 \\
0 & M
\end{array}\right]\left[\begin{array}{l}
\ddot{x}(t) \\
\ddot{y}(t)
\end{array}\right]+\left[\begin{array}{ll}
B_{X X} & B_{X Y} \\
B_{Y X} & B_{Y Y}
\end{array}\right]\left[\begin{array}{l}
\dot{x}(t) \\
\dot{y}(t)
\end{array}\right]+\left[\begin{array}{cc}
C_{X X} & C_{X Y} \\
C_{Y X} & C_{Y Y}
\end{array}\right]\left[\begin{array}{l}
x(t) \\
y(t)
\end{array}\right]=\left[\begin{array}{l}
F_{X}(t) \\
F_{Y}(t)
\end{array}\right]
$$

where $M$ is a mass of the rotor, $F_{X}$ and $F_{Y}$ are forces acting on the journal, and $C$ and $B$ are the stiffness and damping coefficients, respectively. The system is described by two motion equations, and therefore the total order of the system is four. This system may become unstable.

The theory of hydrodynamic bearing is based on a differential equation derived by Osborne Reynolds. Reynolds equation is based on the following assumptions: The lubricant obeys Newton's law of viscosity

\footnotetext{
* Prof. Ing. Jiří Tůma, CSc.: VŠB Technical University of Ostrava, 17. Listopadu 15; 708 33, Ostrava; CZ, milada.kozubkova@vsb.cz

** Ing. Jiř́ Šimek, CSc.: TECHLAB Ltd., Prague, CZ 190 00, Praha 9, CZ, j.simek@techlab.cz

*** Prof. RNDr. Milada Kozubková, CSc.: VŠB Technical University of Ostrava, 17. Listopadu 15; 708 33, Ostrava; CZ, milada.kozubkova@vsb.cz
} 
and is incompressible. The inertia forces of the oil film are negligible. The viscosity $\mu$ of the lubricant is constant and there is a continuous supply of lubricant. The effect of the curvature of the film concerning film thickness is neglected. It is assumed that the film is so thin that the pressure is constant across the film thickness. The shaft and bearing are rigid.
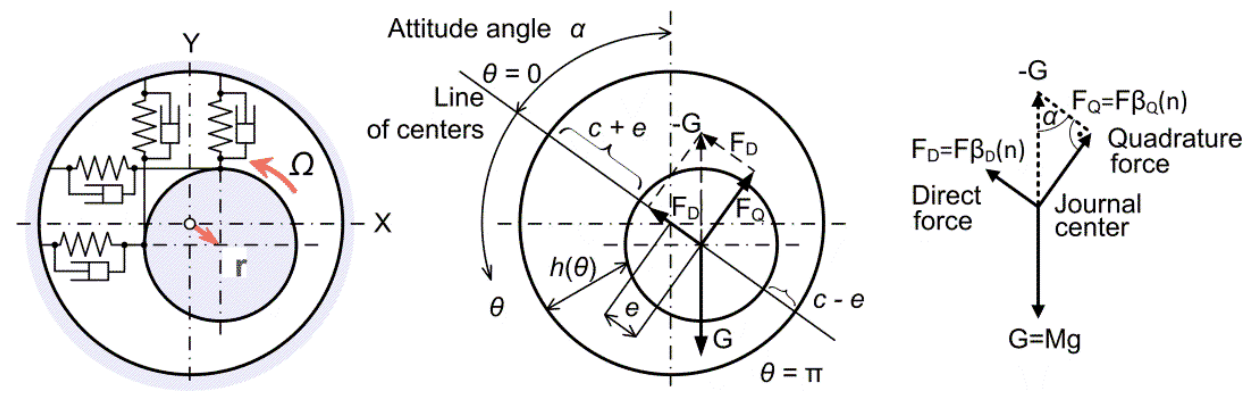

Fig. 1: A cross-section of a hydrodynamic bearing.

\section{Reynolds equation}

Furthermore, it is assumed that the thickness $h$ of the oil film depends on the other two coordinates, namely the coordinate $z$ along the axis of rotation and the location on the perimeter of the journal which is described by the angle $\theta$ as is shown on the right side in Fig. 1. If the radius of the bearing journal is equal to $R$, then the most general version of Reynolds equation for calculation the of the oil pressure distribution $p(\theta, z)$ is as follows (Dwivedy et al., 2006)

$$
\frac{1}{R^{2}} \frac{\partial}{\partial \theta}\left(h^{3} \frac{\partial p}{\partial \theta}\right)+\frac{\partial}{\partial z}\left(h^{3} \frac{\partial p}{\partial z}\right)=6 \mu \Omega \frac{\partial h}{\partial \theta}+12 \mu \frac{\partial h}{\partial t} .
$$

During operation, the journal axis shifts from the centre of the bearing bushing to the distance of $e$, called eccentricity, which is related to a radial clearance $c$. Variable is called an eccentricity ratio $n=e / c$. The film thickness as a function of $\theta$ is as follows

$$
h=c(1+n \cos \theta)
$$

The oil film moves in adjacent parallel layers at different speeds, and shear stress results between them. The oil layer at the surface of the journal moves at the peripheral velocity of the journal while the oil layers at the surface of the bearing bushing don't move (at zero velocity). The surface of the journal moves at a velocity of $U=R \Omega$. Reynolds equation will be solved for the steady state and independence of the pressure distribution on the coordinate of $z$

$$
\frac{d}{d \theta}\left[h^{3} \frac{d p}{d \theta}\right]=6 \mu U R \frac{d h}{d \theta} .
$$

On double integrating, see (Dwivedy et al., 2006), we get

$$
p_{\theta}=\frac{6 \mu U R}{c^{2}} \frac{n(2+n \cos \theta)}{\left(n^{2}+2\right)(1+n \cos \theta)^{2}}+p_{0}=\frac{6 \mu U R}{c^{2}} \beta(\theta, n)+p_{0}
$$

where $p_{0}$ is the second integration constant without any effect on the force excited by the oil pressure. The first integration constant was selected to meet the boundary condition $p_{\theta}(0)=p_{\theta}(2 \pi)$ as is described by Dwivedy et al.. The oil pressure distribution on the journal for $n=0,0.1, \cdots, 0.9$ is shown in Fig. 2 .

The forces acting on the journal in the center of gravity along the bearing length of $L$ can be calculated for the direction of the line of the centers and the perpendicular direction. Force in the direction of the line of centers is denoted as direct force $F_{D}$ while force which is perpendicular to the line of centers is denoted as quadrature force $F_{Q}$. Both these forces balance the gravity force $G$ as is shown in Fig. 1 .

$$
\begin{gathered}
F_{D}=\int_{0}^{2 \pi} p_{\theta} \cos (\pi-\theta) L R \mathrm{~d} \theta=\frac{6 \mu U R^{2} L}{c^{2}} \int_{0}^{2 \pi} \beta(\theta, n) \cos (\pi-\theta) \mathrm{d} \theta=\frac{6 \mu U R^{2} L}{c^{2}} \beta_{D}(n) \\
F_{Q}=\int_{0}^{2 \pi} p_{\theta} \sin (\pi-\theta) L R \mathrm{~d} \theta=\frac{6 \mu U R^{2} L}{c^{2}} \int_{0}^{2 \pi} \beta(\theta, n) \sin (\pi-\theta) \mathrm{d} \theta=\frac{6 \mu U R^{2} L}{c^{2}} \beta_{Q}(n) .
\end{gathered}
$$

A force factor that multiplies the dimensionless functions $\beta_{D}(n)$ and $\beta_{Q}(n)$ can be calculated as follows

$$
F=\frac{6 \mu U R^{2} L}{c^{2}}=6 \pi S G,
$$


where $S=(R / c)^{2} \mu N / P$ is the dimensionless Sommerfeld number, $P=G /(2 L R)$ is the load per unit of projected bearing area and $N$ is the speed of the rotating shaft in rev/s. Note that according to formula (5) the pressure on the part of the journal surface is negative, which is, in fact, a relative negative pressure. Since the pressure distribution is anti-symmetric, without mathematical evidence, it is clear that these formulas can be applied. Only quadrature force acts on the bearing journal and the direct force is zero $F_{D}=0, F_{Q}>0$ as is shown on the right panel in Fig. 3.

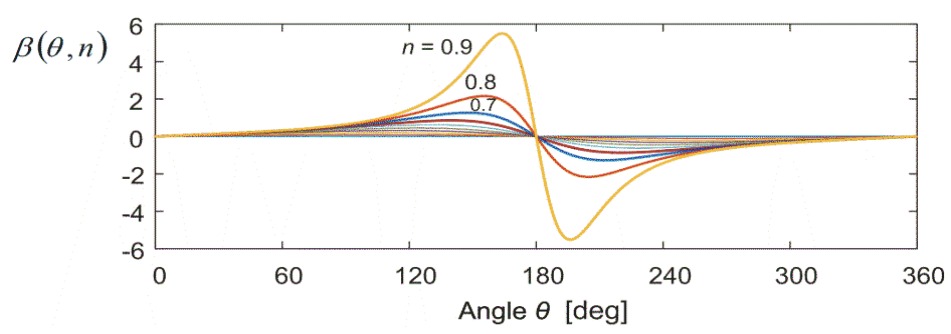

Fig. 2: Pressure distribution along the angular coordinate.
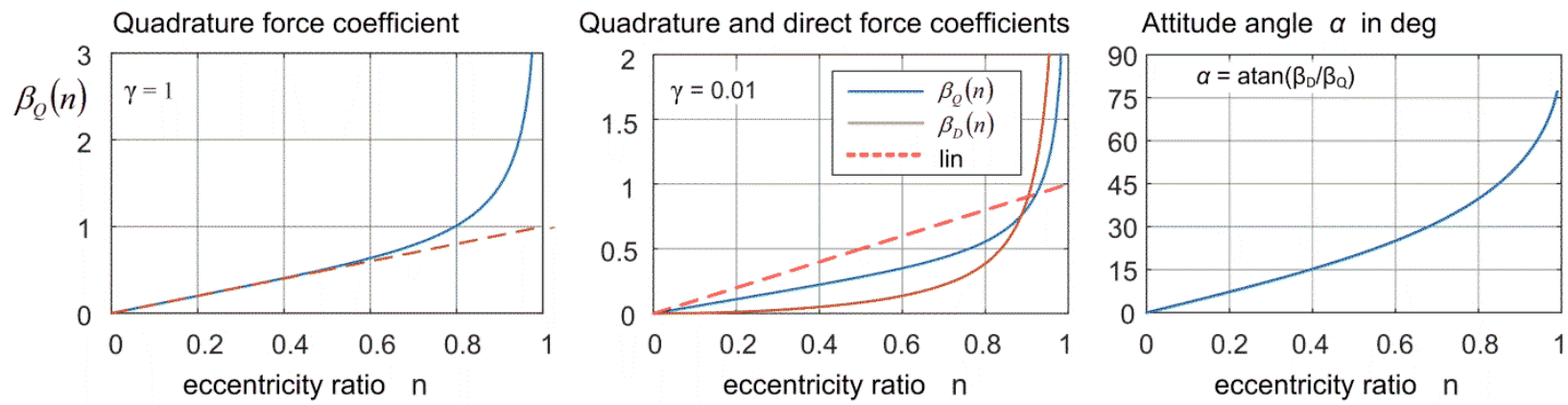

Fig. 3: Dependence of the direct and quadrature force on the eccentricity ratio.

This case can only theoretically arise in a fully flooded plain bearing with a vertical axis. The balance of forces $F_{D}, F_{Q}$ and $G$ allows to calculate an attitude angle $\alpha$ as $\operatorname{atan}\left(\beta_{D} / \beta_{Q}\right)$, see Fig. 3. The presence of direct force can be explained eg. by the cavitations but the mathematical model is more complicated (Ferfecki et al., 2007). The magnitude of the negative pressure for $\pi<\theta<2 \pi$ is multiplied by a factor $\gamma$, therefore the total force is given by the sum of integrals (6) as follows $\int_{0}^{\pi}(\ldots) \mathrm{d} \theta+\gamma \int_{\pi}^{2 \pi}(\ldots) \mathrm{d} \theta$. The effect of negative pressure reduction is demonstrated in Fig. 3. Negative pressure is limited to $1 \%$ of the magnitude of positive pressure for the angle interval of $0<\theta<\pi$. The formulas for the calculation of the quadrature and direct forces contain the same factor $F=6 \mu U R^{2} L / c^{2}$ and hence the dependence on the peripheral speed $U$ and therefore on the rotor angular velocity. The coefficients $\beta_{Q}(n)$ and $\beta_{D}(n)$ differ considerably. The diagrams confirm the linearity of the quadrature and direct force to eccentricity ratio up to 0.6 . The $\beta_{Q}(n)$ and $\beta_{D}(n)$ coefficients can be approximated in this range as a linear function

$$
\beta_{Q}(n) \approx q c n=q e \text { and } \beta_{D}(n) \approx d c n=d e
$$

where $q$ and $d$ determine the quadrature stiffness $C_{Q}=6 \mu U R^{2} L / c^{2} \times q$ and the direct stiffness $C_{D}=6 \mu U R^{2} L / c^{2} \times d$. The stiffness in the directions of the Cartesian coordinates $x(t)=-e \sin \alpha$ and $y(t)=\mathrm{e} \cos \alpha$ is influenced by the attitude angle $\alpha$

$$
\left[\begin{array}{c}
-C_{D} e \sin \alpha+C_{Q} e \cos \alpha \\
C_{Q} e \sin \alpha+C_{D} e \cos \alpha
\end{array}\right]=\left[\begin{array}{c}
C_{D} x(t)+C_{Q} y(t) \\
-C_{Q} x(t)+C_{D} y(t)
\end{array}\right]=\left[\begin{array}{cc}
C_{D} & C_{Q} \\
-C_{Q} & C_{D}
\end{array}\right]\left[\begin{array}{l}
x(t) \\
y(t)
\end{array}\right]
$$

This chapter was focused on the derivation of the formulas for the calculation of the direct and quadrature force acting on eccentric journal axis of the plain bearing. The result is the stiffness matrix in Eq. (1). The damping matrix was not derived. The numerical solution of Reynolds equation allows you to respect the pressure distribution along the journal axis, i.e. also in dependence on the $z$ coordinate. Authors of the paper have a test rig (see the right panel in Fig. 4) with the following parameters. The span of bearing pedestals is of $200 \mathrm{~mm}$, the journal diameter is of $30 \mathrm{~mm}$, the radial clearance is of $55 \mu \mathrm{m}$, and the lengthto-diameter ratio is equal to about 0.77 . Pertinent stiffness and damping coefficients are obtained by solving Reynolds equation providing that the journal performs the small harmonic motion in a neighborhood of its equilibrium position as shown in Fig. 4 (Šimek). Notice the magnitude asymmetry of 
the cross-coupled stiffness as opposed to (9). Even the numerical solution of the Reynolds equation is not perfect because these coefficients which are used for simulation do not lead to the stable journal rotation.
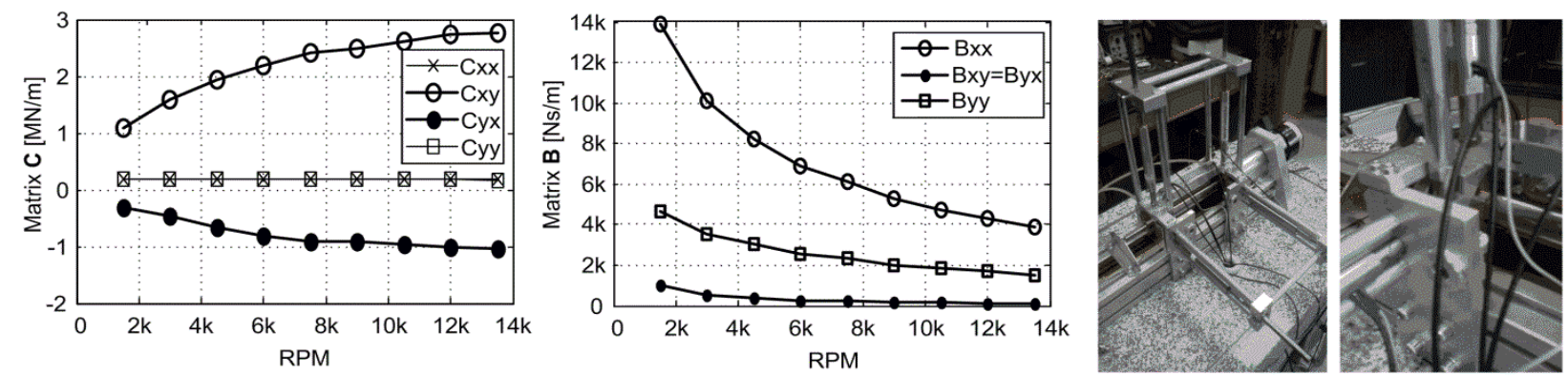

Fig. 4: Real stiffness and damping matrices according to the Reynolds model [10] and test rig.

\section{Muszynska model}

The motion equation of the rotor with the journal bearing in coordinates $x$ and $y$ was designed by Muszynska (1986). Compared to Eq. (1) the stiffness and damping matrices are designed in such a way that the oil film is replaced by a spring and a damper that rotates at an angular velocity $\lambda \Omega$

$$
\left[\begin{array}{cc}
M & 0 \\
0 & M
\end{array}\right]\left[\begin{array}{c}
\ddot{x}(t) \\
\ddot{y}(t)
\end{array}\right]+\left[\begin{array}{ll}
D & 0 \\
0 & D
\end{array}\right]\left[\begin{array}{l}
\dot{x}(t) \\
\dot{y}(t)
\end{array}\right]+\left[\begin{array}{cc}
K & D \lambda \Omega \\
-D \lambda \Omega & K
\end{array}\right]\left[\begin{array}{l}
x(t) \\
y(t)
\end{array}\right]=\left[\begin{array}{l}
F_{X}(t) \\
F_{Y}(t)
\end{array}\right]
$$

where $K$ and, $D$ are specifying proportionality of stiffness and damping to the relative position of the journal center displacement vector, $\lambda$ is a dimensionless parameter, which is slightly less than 0.5 . The cross-coupled stiffness $D \lambda \Omega$ according to the Muszynska model corresponds to the expression $6 \mu U R^{2} L / c^{2} q$. The direct stiffness $K$ is orderly less than the cross-coupled stiffness; however, the analytical calculation shows the dependence on the rotational speed. The sum of direct and quadrature forces must compensate for the gravitational force that does not depend on the speed of rotation. The suitability of this model is confirmed by Cavalca (2014).

\section{Conclusions}

The calculation of the fluid force in the steady-state acting on the bearing journal using the Reynolds equation shows that the stiffness matrix corresponds to the stiffness matrix according to the Muszynska model. The solution of the Reynolds equation shows that, unlike the Muszynska model, the diagonal entries of the stiffness matrix depend on the rotational speed of the rotor. The conclusions apply only to the range of magnitudes in which the formulas can be linearized. The theoretical models are always unreliable and require comparative measurement on the test rig.

\section{Acknowledgement}

Authors thank to the European Regional Development Fund in the Research Centre of Advanced Mechatronic Systems project No. CZ.02.1.01/0.0/0.0/16_019/0000867 within the Operational Programme Research, Development and Education and also thank to the R\&D in the project No. SP2018/123.

\section{References}

Dwivedy, S. K. and Tiwari, R. (2006) Dynamics of Machinery, A Lecture Notes, All India Council of Technical Education, March 2006.

Ferfecki, P., Zapoměl, J., and Kozánek, J. (2007) Analysis of the vibration attenuation of rotors supported by magnetorheological squeeze film dampers as a metaphysical finite element problem, Advances in Engineering Software 2017, 104, pp. 1-11.

Mendes R.U. and Cavalca K. L. (2014) On the Instability Threshold of Journal Bearing Supported Rotors. International Journal of Rotating Machinery, Volume 2014, Article ID 351261.

Muszynska A. (1986) Whirl and whip - rotor/bearing stability problems. Journal of Sound and Vibration, Vol. 110, Issue 3, 1986, p. 443-462.

Šimek, J., Dynamic properties of plain journal bearings. [Online.] available:www.techlab.cz.

Tůma, J., Šimek, J., Mahdal, M., Škuta, J. and Wagnerová, R. (2017) Actively Controlled Journal Bearings, in: Proc. 12th SIRM Conference, Graz, Österreich, 15. - 17. Februar 2017, pp 71-82. 